\title{
Vitamin K2-induced antitumor effects via cell-cycle arrest and apoptosis in gastric cancer cell lines
}

\author{
HIROMI TOKITA, AKIHIKO TSUCHIDA, KEISUKE MIYAZAWA, KAZUMA OHYASHIKI, SOH KATAYANAGI, \\ HIDEO SUDO, MASANOBU ENOMOTO, YU TAKAGI and TATSUYA AOKI
}

\begin{abstract}
Third Department of Surgery and First Department of Internal Medicine, Tokyo Medical University, Tokyo, Japan
\end{abstract}
Received September 12, 2005; Accepted October 24, 2005

\begin{abstract}
Vitamin K2 (VK2) has a growth inhibitory effect on various types of cancer cells in vitro, and its efficacy has been demonstrated in clinical applications in a number of patients with leukemia and hepatocellular carcinoma. In this study, the effect of cell growth inhibition and apoptosis induction and the concomitant use of an anticancer agent by VK2 (menaquinone: MK4), on gastric cancer cell lines were examined. When 4 kinds of gastric cancer cells (KATO III, MKN7, MKN74 and FU97) were exposed to MK4, the cell growth was inhibited in an MK4 dose-dependent manner. Morphologically, apoptosis induced by MK4 was recognized in FU97, but only a slight number of apoptotic images was recognized in other cell lines. On the contrary, in all the cell lines, the percentage of APO2.7 positive cells increased significantly in the MK4-treated group as compared to the controls. Caspase-3 activity increased significantly in KATO III and FU97 as compared to the controls, while no significant differences were noted in MKN7 or MKN74. Moreover, in all the cell lines, the percentage of G0/G1-phase cells ( $70 \%$ in KATO III and FU97, and $\geq 80 \%$ in MKN7 and MKN74) increased in comparison to the controls, suggesting that cellcycle arrest had occurred. All of the gastric cancer cell lines were given MK4 in different concentrations and two kinds of anticancer agent, with the result that cell growth was inhibited by the anticancer agent in a dose-dependent manner when it was given with MK4 in concentrations of up to $10 \mu \mathrm{M}$. In conclusion, our results demonstrate that the effect of MK4 on apoptosis and cell-cycle arrest differs in differentiated (MKN7, MKN74) and undifferentiated (KATO III, FU97) gastric cancer cell lines, and that MK4 alone or with anticancer agents has an antitumor effect on gastric cancer cell lines.
\end{abstract}

Correspondence to: Dr Akihiko Tsuchida, Third Department of Surgery, Tokyo Medical University, 6-7-1 Nishi-shinjuku, Shinjukuku, Tokyo 160-0023, Japan

E-mail: akibobo@hotmail.com

Key words: gastric cancer, vitamin K2, chemoprevention, cell-cycle arrest, apoptosis

\section{Introduction}

There are 13 types of vitamin $\mathrm{K}$-dependent proteins in the human body, including blood coagulant factors (II, VII, IX and $\mathrm{X}$ factors), anticoagulant factors (protein $\mathrm{C}$, protein $\mathrm{S}$ ) and bone protein (osteocalcin, matrix Gla protein) (1). Vitamin K (VK) acts as a coenzyme that reacts to modify a specific residual radical of glutamic acid found along the amino end of these precursor proteins into $\gamma$-carboxy glutamic acid (Gla conversion). Of the 3 types of VK, VK1 (phytonadione) and VK2 (menaquinone) are found in nature and VK3 (menadione) is synthesized chemically (1). For a long time after it was discovered in 1929, VK was regarded as an essential nutrient for hemostasis. In the 1980's, it was evident that VK3 has the ability to inhibit cell growth and to induce apoptosis, but it has not yet been applied clinically because of its hepatic toxicity.

VK2 is named menaquinone (MK)-n according to the number (n) of isoprenoid radicals that make up its side chain, and 14 of these (MK1-MK14) are found in nature. In recent years, it has been reported that VK2 has a cell growth inhibitory effect and a differentiation inducing effect against tumor cells of various leukemias and hepatocellular carcinomas (2-12). The mechanisms of these antitumor effects are believed to differ from Gla conversion of amino acids, but the details have not yet been elucidated. Clinically, there are also a number of reports on the efficacy of VK2 against such things as myelodysplastic syndrome, leukemia and hepatocellular carcinoma (13-16). Generally speaking, the strength of this growth inhibitory effect is found to be in the sequence: VK3, VK2 and VK1 $(7,9,11)$. In several reports, the antitumor effect of VK3 was lost by adding catalase and, for this reason, it is suspected that VK3 damages cancer cells by influencing the oxidation-reduction cycle. On the contrary, the antitumor effect of VK2 is not inhibited by catalase, so it is conjectured that the action mechanisms of the two are different $(9,17,18)$. An antitumor effect is also reported for the VK2 side chains, geranylgeraniol (GGO) and geranylfarnesol $(\mathrm{GFO})(3,12,19)$. In examining the effects of VK2, the influences of side chains must also be considered.

There are a number of reports on the antitumor effect of VK2 against leukemia cells and hepatocellular carcinoma cells, but virtually no studies have been done on the effect against gastric cancer cells. In the present study, therefore, an oral vitamin K2 drug (menaquinone-4: MK4), which is widely 
used as a therapeutic agent against osteoporosis, was used for in vitro examination of the influence of VK2 on 4 kinds of gastric cancer cells lines in terms of cell growth inhibition, apoptosis induction and the effects of the concurrent use of anticancer drugs. MK4 differs from anticancer drugs in that it produces few serious side effects. Its safety for long-term intake by the elderly has been established (20), and it is expected to be effective in cancer treatment or chemoprevention.

\section{Materials and methods}

Reagents. VK2 (menaquinone-4: MK4) was synthesized and supplied by Eisai Co. Ltd. (Tokyo, Japan).

Cell lines. All cell lines originated from human gastric cancer tissues. KATO III (signet ring cell carcinoma), MKN7 (welldifferentiated tubular adenocarcinoma), MKN74 (moderatelydifferentiated tubular adenocarcinoma, liver metastasis) and FU97 (poorly-differentiated adenocarcinoma, AFP production) cells were provided by the Japanese Collection Research Bioresources Cell Bank (Tokyo, Japan). KATO III cells were cultured in RPMI-1640 medium with Eagle's minimal essential medium and 10\% FBS. MKN7 and MKN74 cells were cultured in RPMI-1640 medium with 10\% FBS. FU97 cells were cultured in Dulbecco's modified Eagle's medium (4.5 g/l glucose) with $10 \%$ fetal calf serum and $10 \mathrm{mg} / \mathrm{l}$ insulin. All cells were cultured in an atmosphere of $5 \% \mathrm{CO}_{2}$ in air at $37^{\circ} \mathrm{C}$.

Growth inhibitory effects of MK4. After the cell count was adjusted to $1 \times 10^{4}$ cells $/ 100 \mu 1$ and MK4 was added in various concentrations, specimens were placed on a 96-well tissue culture plate (BD Biosciences, Bedford, MA, USA) and incubated for $96 \mathrm{~h}$. A cell counting kit (Dojindo Laboratories, Kumamoto, Japan) was used to determine the surviving cell count after $96 \mathrm{~h}$. Cells cultivated under the same conditions but without the addition of MK4 were taken as controls, and the percentage of cells as opposed to the controls was calculated as the relative cell number.

Morphological appearance of gastric cancer cells treated with $M K 4$. A control group and a group to which $50 \mu \mathrm{M}$ of MK4 had been added were prepared and the cell morphology, $96 \mathrm{~h}$ after incubation, was observed. KATO III were suspended cells, and a specimen was created by adhering the cells to a preparation using Shandon Cytospin 2 (Shandon, Pittsburgh, PA, USA). The cell morphology was then observed under Giemsa staining. MKN7 were adhesive cells and those cultivated on chamber slides underwent Giemsa staining without modification and were examined microscopically. MKN74 and FU97 were adhesive cells and the morphology of those cultivated on chamber slides was observed under a phase contrast microscope. In addition, cells were treated with trypsin and separated, and individual cell morphologies were observed.

Flow cytometric detection of apoptotic cells using APO2.7 monoclonal antibody $(\mathrm{mAb})$. Cells undergoing apoptosis were assessed by flow cytometry using APO2.7 mAb. The cells were washed twice with PBS containing 5\% FBS. Then,
$1 \times 10^{5}$ cells suspended in $0.5 \mathrm{ml}$ of PBS containing 5\% FBS and $0.02 \% \mathrm{NaN}_{3}$ were incubated with one or two of the following $\mathrm{mAb}$ for $30 \mathrm{~min}$ at $4^{\circ} \mathrm{C}$; the mouse FITC-conjugated $\mathrm{mAb}$, APO2.7 (clone 2.7 A6A3) for detecting 7A6 antigen expressed by cells undergoing apoptosis, was obtained from Immunotech (Marseille, France). Immunofluorescence was analyzed by flow cytometry using an EPICS XL2 flow cytometer (Coulter Japan, Tokyo, Japan). The percentage of APO2.7 positive cells in gastric cancer cells with $50 \mu \mathrm{M}$ of MK4 was counted and compared with controls.

Assessment of caspase-3 activity in MK4-induced apoptosis. Caspase-3 activity was examined by flow cytometry using a substrate reagent kit containing PhiPhiLux-G6D2, a rhodaminecontaining specific substrate with amino-acid sequence treatment (Oncolmmunin, College Park, MD, USA). Briefly, the cells treated with/without MK4 were washed with PBS and incubated with a substrate reagent at $37^{\circ} \mathrm{C}$ for $60 \mathrm{~min}$. The fluorescence of the profluorescent substrate cleaved by activated caspase- 3 was analyzed by flow cytometry at the FL2 channel of a BD instrument with excitation at $488 \mathrm{~nm}$. Caspase- 3 activity in gastric cancer cells with $50 \mu \mathrm{M}$ of MK4 was measured and compared with controls.

Cell-cycle analysis. Cells were fixed and stained with a solution containing 1\% propidium iodide (Sigma), $100 \mu \mathrm{g} / \mathrm{ml}$ digitonin, $0.01 \% \mathrm{NaN}_{3}, 200 \mu \mathrm{g} / \mathrm{ml} \mathrm{RNase}$ (Sigma) and $2.5 \%$ FCS for $10 \mathrm{~min}$ at room temperature. Cells were analyzed by flow cytometry, and the populations of the G0/G1, S, and G2/M phases were calculated using a cell-cycle analysis program, MultiCycle AV (Phoenix Flow Systems, San Diego, CA, USA).

Effect of combined treatment of anticancer drugs with MK4. Two anticancer agents, 5-fluorouracil (5-FU; Kyowa Hakko Kogyo Co., Tokyo, Japan) and docetaxel (TXT; Sanofi Aventis, Paris, France), were added together with MK4 at various concentrations to culture medium containing 4 kinds of gastric cancer cell and incubated for $96 \mathrm{~h}$. The surviving cell count was determined using a cell counting kit (Dojindo Laboratories). Cells cultivated under the same conditions but without the addition of MK4 were taken as controls, and the percentage of cells as opposed to the controls was calculated as the survival rate. Results were calculated from the mean values and the standard deviation (SD) of data on 5 plates.

Statistical analysis. All data are shown as mean \pm SD. Comparisons between two groups were assessed using the Student's t-test. A p-value of less than 0.05 was considered to indicate a statistically significant difference.

\section{Results}

Growth inhibitory effects of MK4. We examined the growth of 4 kinds of gastric cancer cells in the presence of various concentrations of MK4 and estimated $\mathrm{ID}_{50}$. As shown in Fig. 1, a 96-h exposure to MK4 in all cell lines resulted in inhibition of cell growth in a dose-dependent manner. ID $_{50}$ were $35 \mu \mathrm{M}$ in KATO III, $50 \mu \mathrm{M}$ in MKN7, $25 \mu \mathrm{M}$ in MKN74 and $35 \mu \mathrm{M}$ in FU97, respectively. 

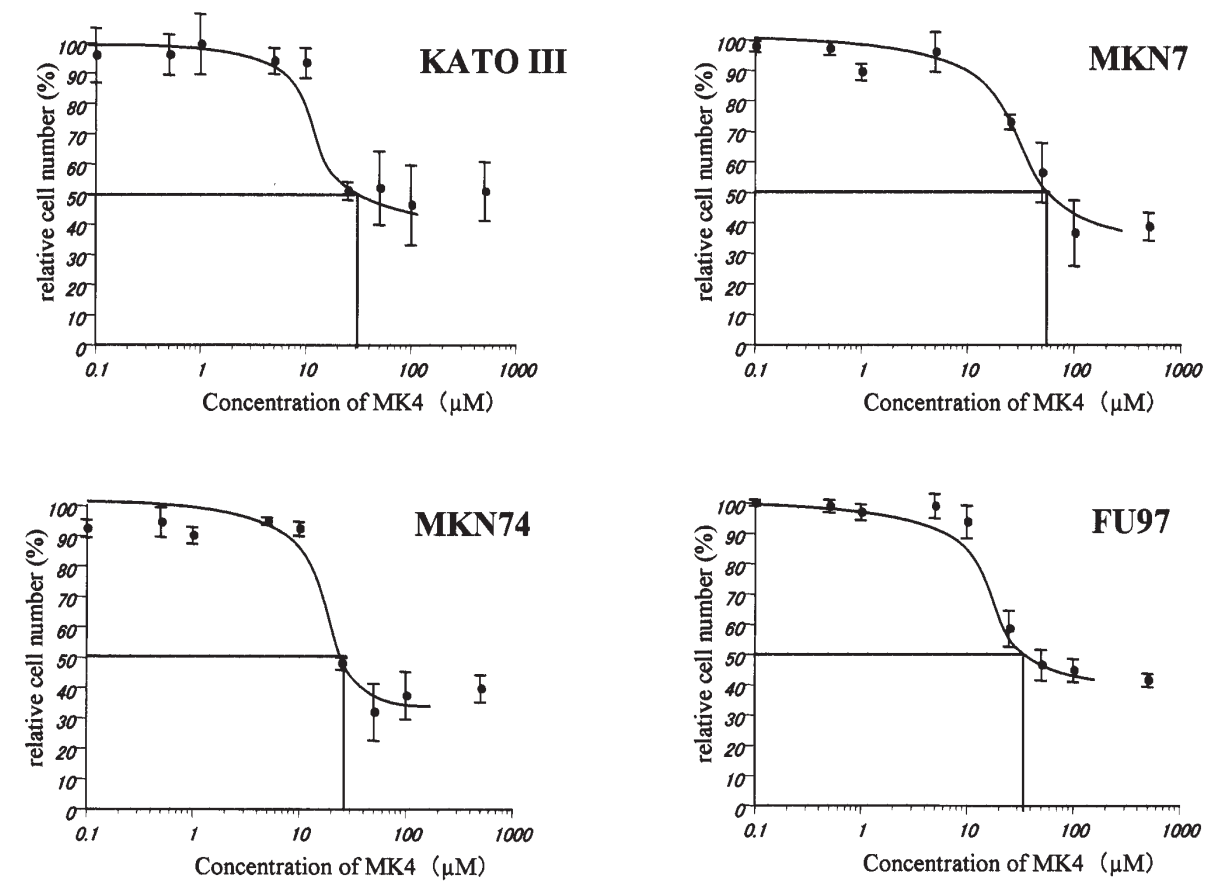

Figure 1. Growth inhibition of gastric cancer cell lines in the absence or presence of various concentrations of MK4.

A

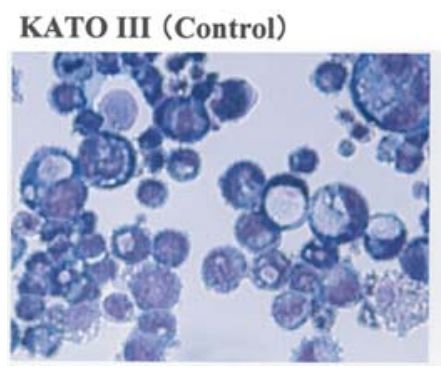

C

\section{MKN74 (Control)}
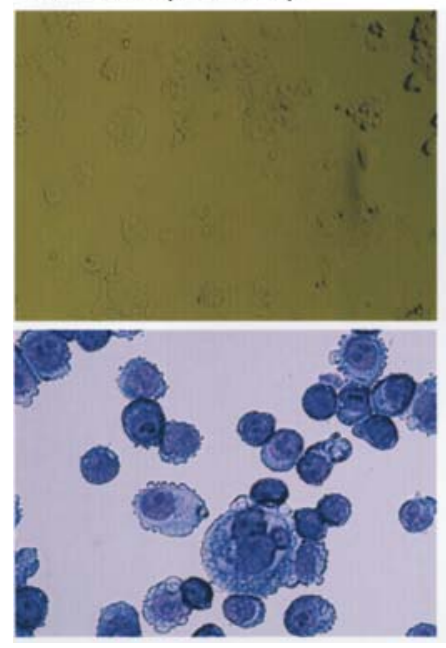

(MK4)

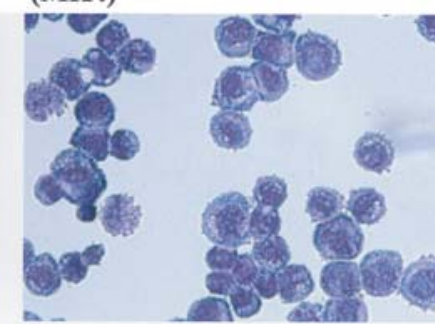

(MK4)
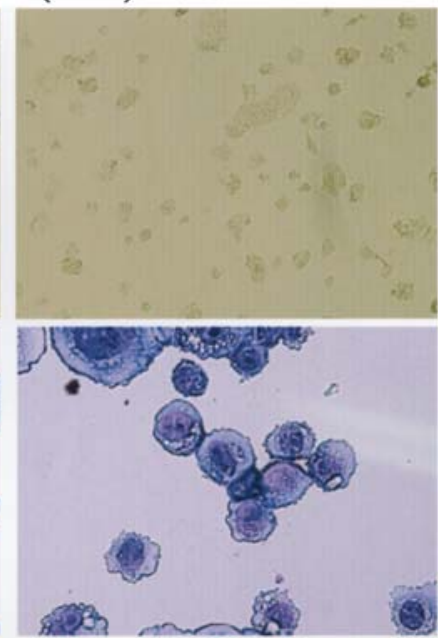

Figure 2. Morphological changes after 96-h exposure to MK4. (A) KATO III (Giemsa staining), (B) MKN7 (upper, phase contrast microscopic view; lower, Giemsa staining), (C) MKN74 (the same as MKN7) and (D) FU97 (the same as MKN7) (original magnification, $\mathrm{x} 400$ ).

\section{B}

MKN7 (Control)
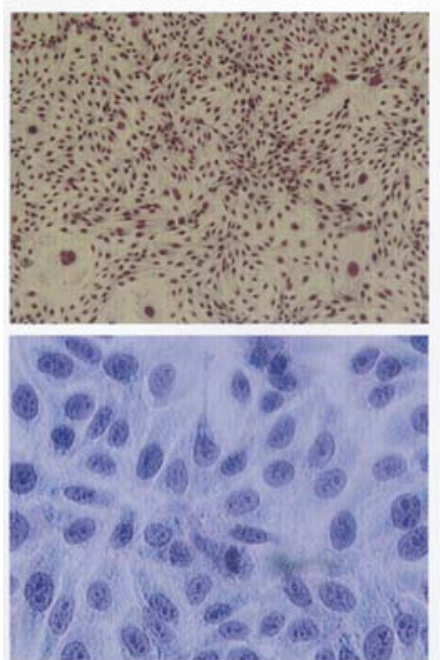

D

FU97 (Control)

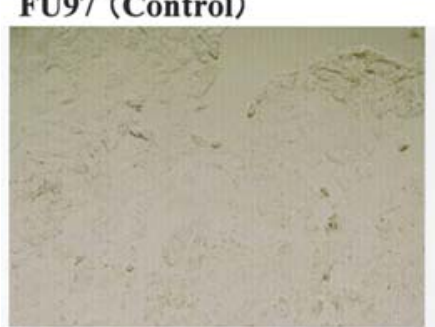

(MK4)
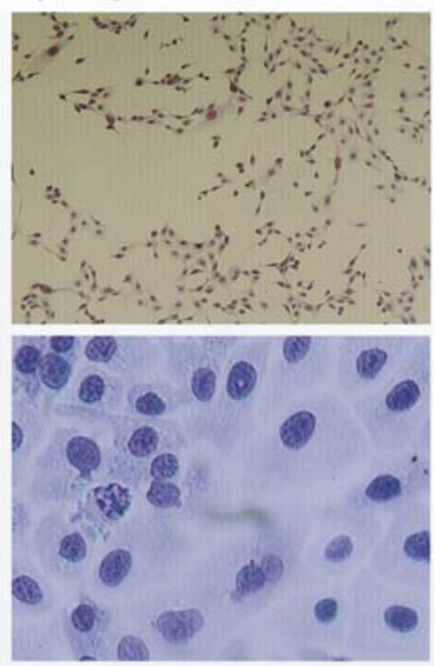

(MK4)
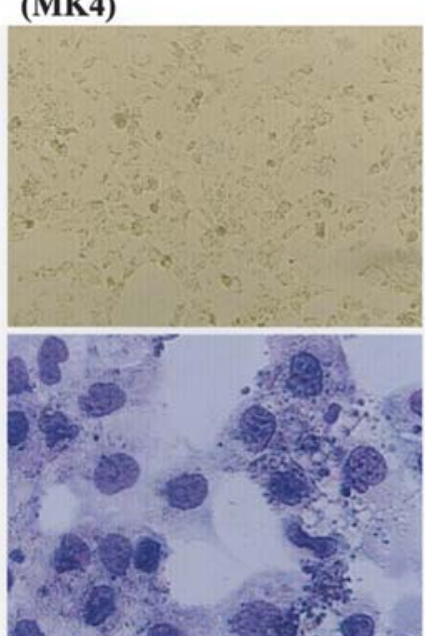
A

KATO III
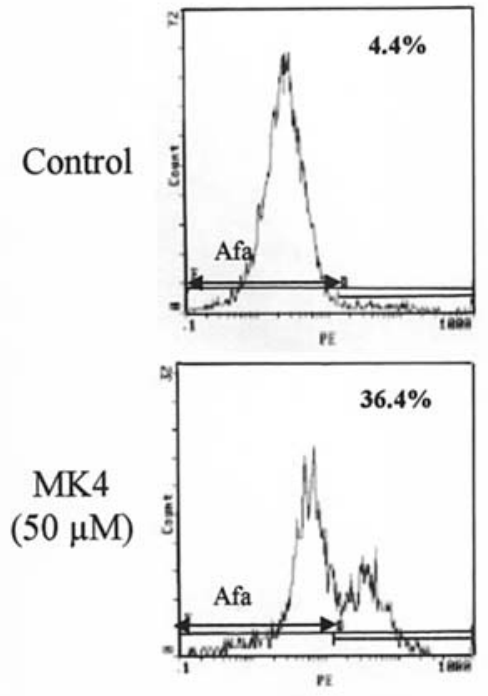

MKN7
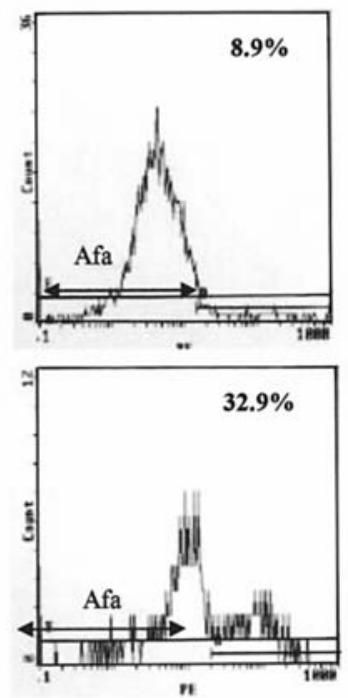

MKN74
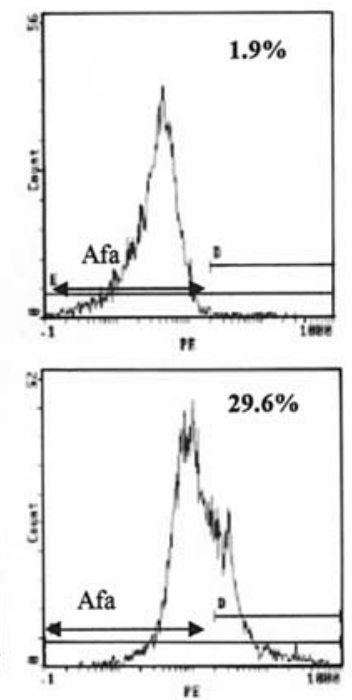

FU97
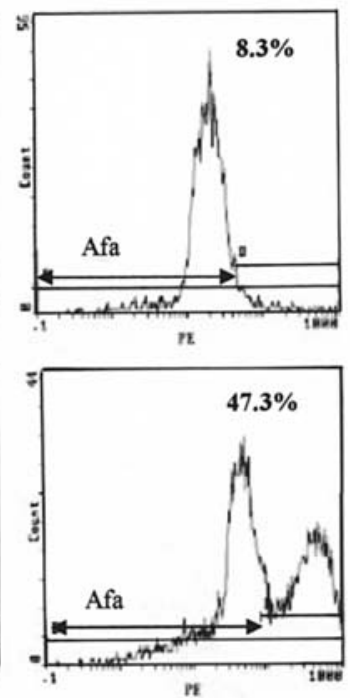

B KATO III MKN7 MKN74

FU97
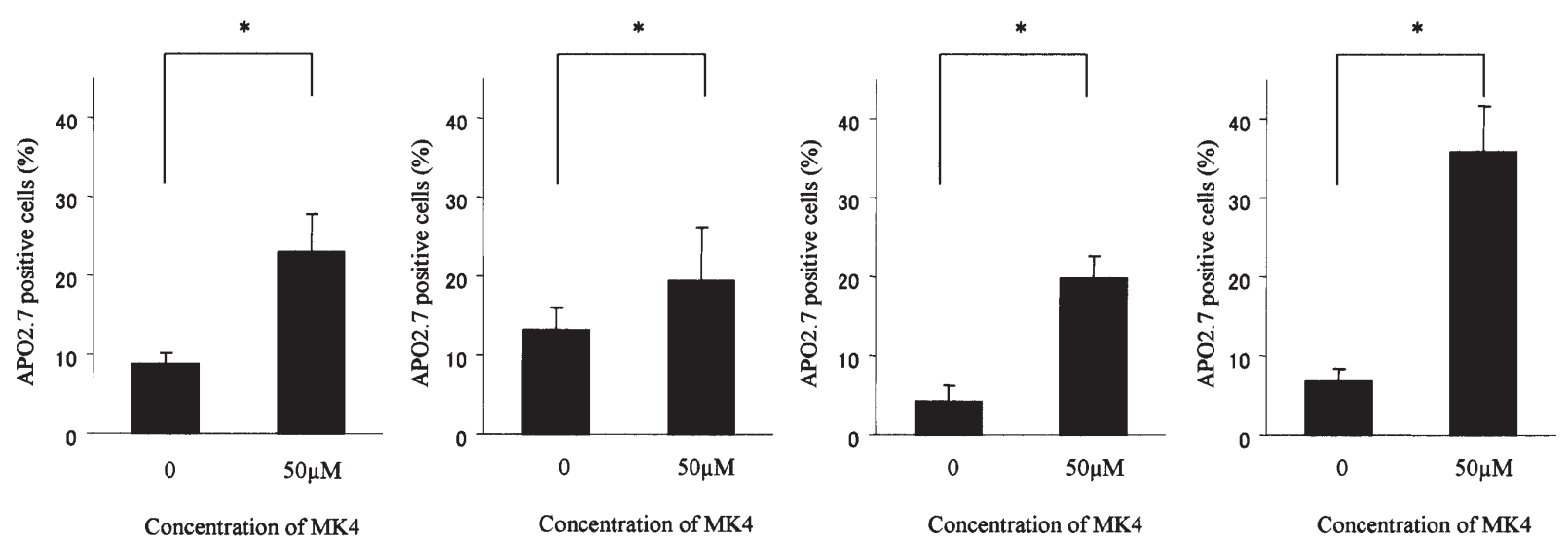

Figure 3. Flow cytometric detection of MK4-induced apoptosis. (A) Representative results of cells undergoing apoptosis using APO2.7 mAb. Numbers are the percentages of cells positive for APO2.7 mAb. (B) Summarized results of cells undergoing apoptosis using APO2.7 mAb.

Morphological appearance of gastric cancer cells treated with MK4. We examined the microscopic appearance of gastric cancer cells after 96-h treatment with MK4 compared with controls. KATO III underwent Giemsa staining. Because KATO III are suspended cells that originate from signet ring cell carcinoma, they contain mucus in their interior when the cells mature. It was noted that, in the MK4-treated group, the percentage of cells with mucus in their interior diminished in comparison to the controls. Nevertheless, virtually no findings were recognized as being indicative of apoptosis, such as apoptotic bodies, nuclear shrinkage or fragmentation (Fig. 2A).

MKN7 was propagated on preparation and observed. In a slightly enlarged image from phase contrast microscopy, it was found that control group cell lines grew confluently, while cell growth in the MK4-treated group was clearly inhibited. Under Giemsa staining, nuclear fragmentation was observed in only a small number of cells in the MK4 group. Overall, however, there were no findings indicative of apoptosis and only a decrease of $\mathrm{N}$ : C ratio was noted (Fig. 2B).
In observations of MKN74 under phase contrast microscopy, there was cell luster and thickness in the control group and regions where some of the cells stratified and grew. In the MK4-treated group, however, changes in the granular condition of cytoplasm were recognized and the impression was gained that the cytoplasm is thin. Under Giemsa staining, in the MK4-treated group, as in MKN74, no findings indicative of apoptosis were evident, and a decrease of $\mathrm{N}: \mathrm{C}$ ratio was noted in many of the cells (Fig. 2C).

In observations of FU97 under phase contrast microscopy, as in the case of MKN74, the cells were found to be growing with luster and thickness. Under Giemsa staining, the individual cell morphology became irregular compared to the control group and the apoptotic body was markedly recognized, indicating MK4-induced apoptosis (Fig. 2D).

Flow cytometric detection of apoptotic cells using APO2.7 monoclonal antibody. APO2.7 is a novel monoclonal antibody which is raised against the $38-\mathrm{kDa}$ mitochondrial membrane 
A
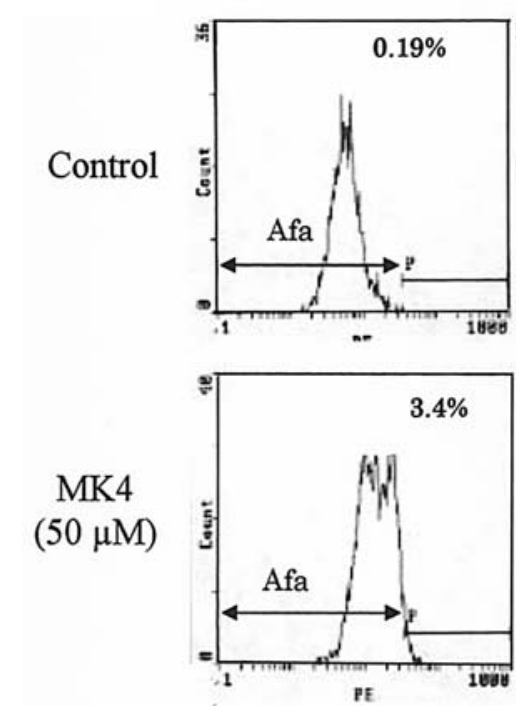

B

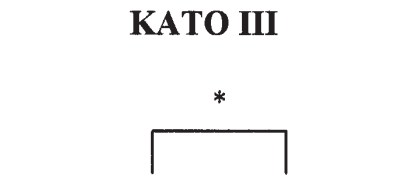

MKN7
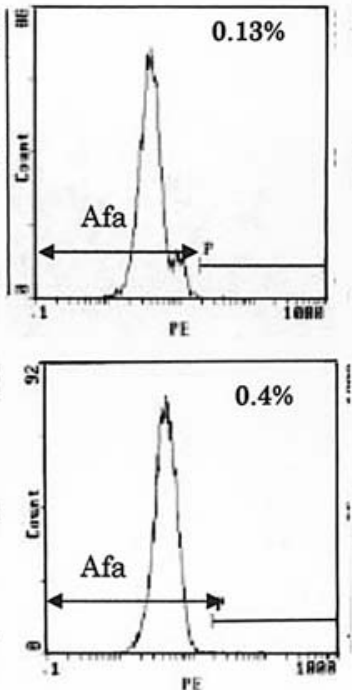

MKN7
MKN74
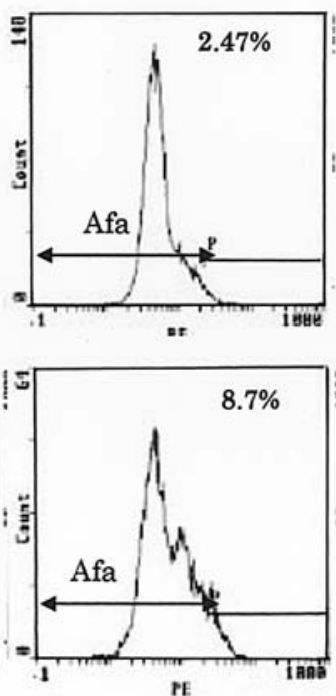

MKN74
FU97
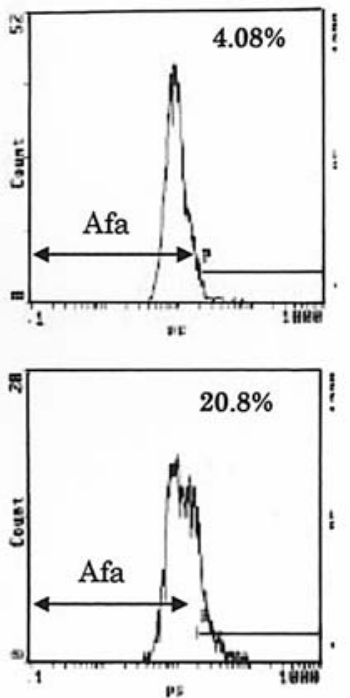

FU97

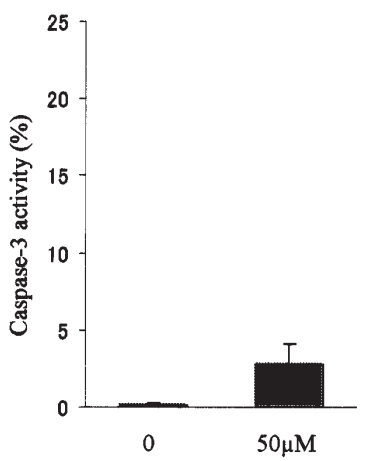

Concentration of MK4

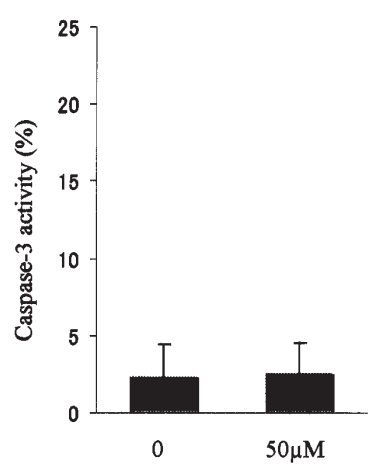

Concentration of MK4

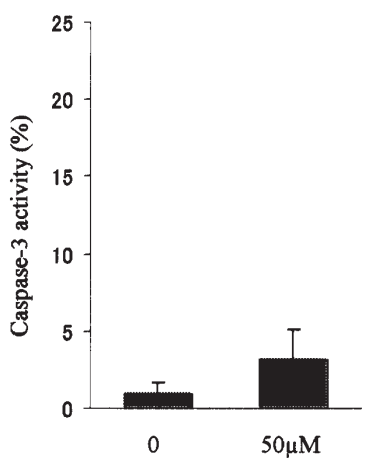

Concentration of MK4

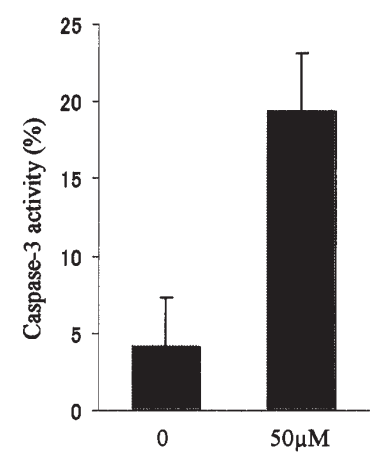

Concentration of MK4

Figure 4. Flow cytometric detection of caspase-3 activity. (A) Representative results. (B) Summarized results.

protein (7A6 antigen) specifically expressed by cells undergoing apoptosis. In all gastric cancer cells, the percentage of APO2.7 positive cells was significantly increased after a 96-h exposure to MK4 compared with controls (Fig. 3).

Assessment of caspase-3 activity in MK4-induced apoptosis. MK4 $(50 \mu \mathrm{M})$ was added to 4 kinds of gastric cancer cell and caspase-3 activity was measured after $72 \mathrm{~h}$. In KATO III and FU97, caspase-3 activity increased significantly as compared to the controls, but no significant difference was recognized in MKN7 and MKN74 (Fig. 4).

Cell-cycle analysis. MK4 (50 and $100 \mu \mathrm{M})$ were added to 4 kinds of gastric cancer cell and the cell cycle was analyzed after $48 \mathrm{~h}$. In all of the cancer cells, the percentage of G0/G1phase cells was elevated compared to the controls, suggesting that cell-cycle arrest had occurred (Fig. 5). The percentage of G0/G1 phase was about 70\% in KATO III and FU97, while it was $80 \%$ or more in MKN7 and MKN74.
Effect of combined treatment of MK4 with anticancer drugs. To 4 kinds of gastric cancer cell, 25, 50 and $100 \mu \mathrm{M}$ of MK4 were added together with 5-FU of various concentrations and survival cell counts were calculated. For all the cancer cells, with 5-FU concentration of up to $10 \mu \mathrm{g} / \mathrm{ml}$, cell growth was inhibited in a dose-dependent manner in the MK4-added group as compared to the controls. At a 5-FU concentration of over $10 \mu \mathrm{g} / \mathrm{ml}$, no differences in survival cell count were recognized in any of the cancer cell types (Fig. 6). Similarly, with a docetaxel concentration of up to $10 \mu \mathrm{g} / \mathrm{ml}$, cell growth was inhibited in a dose-dependent manner in the MK4-added group as compared to the controls. At a docetaxel concentration of over $10 \mu \mathrm{g} / \mathrm{ml}$, no differences in survival cell count were recognized in any of the cancer cell lines (Fig. 7).

\section{Discussion}

We set out to determine whether VK2 has antitumor effects, including the inhibition of cell growth, the induction of 
KATO III

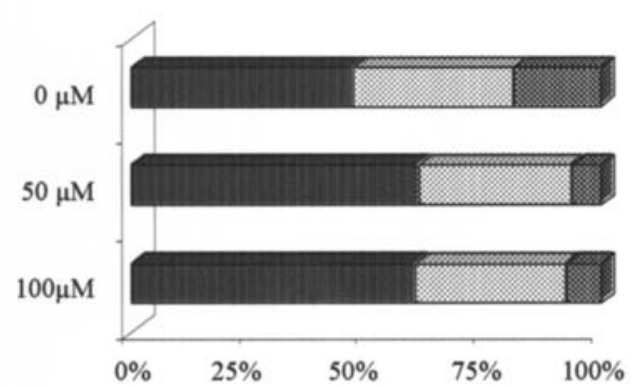

MKN74

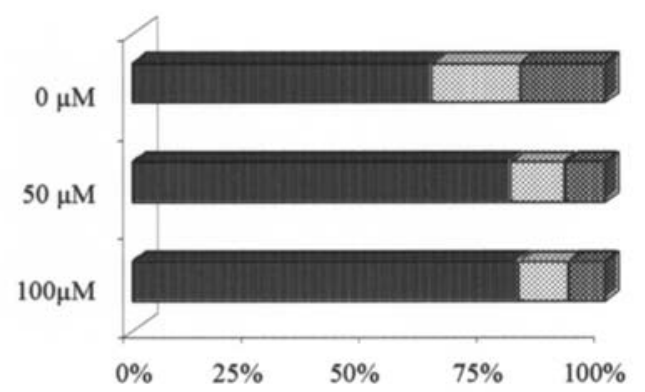

MKN7

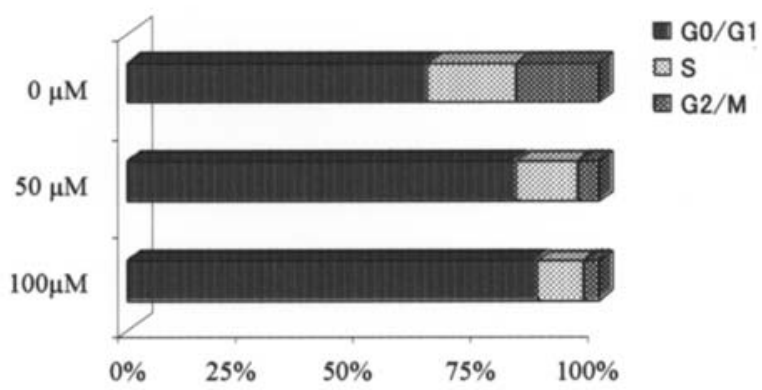

FU97

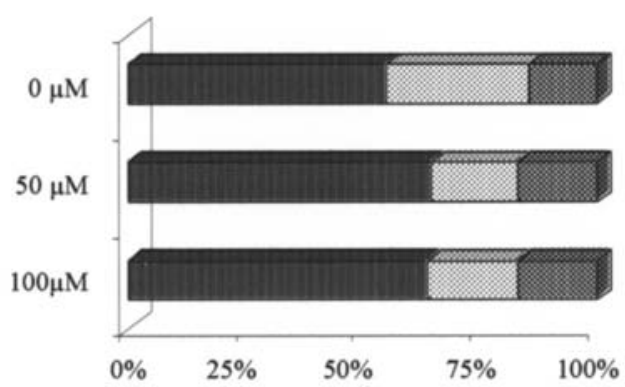

Figure 5. Cell-cycle analysis of gastric cancer cell lines in the absence or presence of various concentrations of MK4.
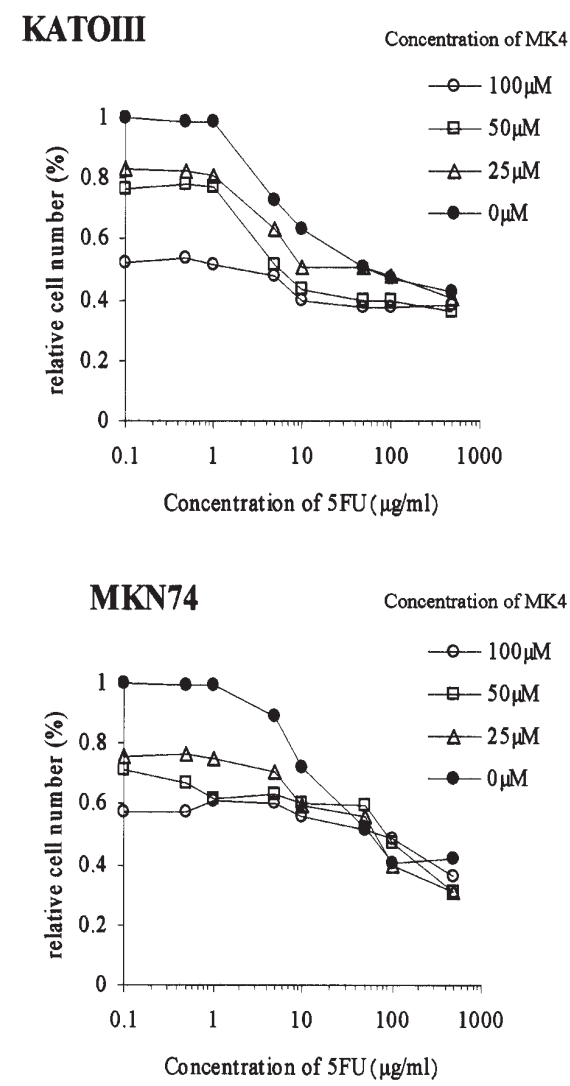
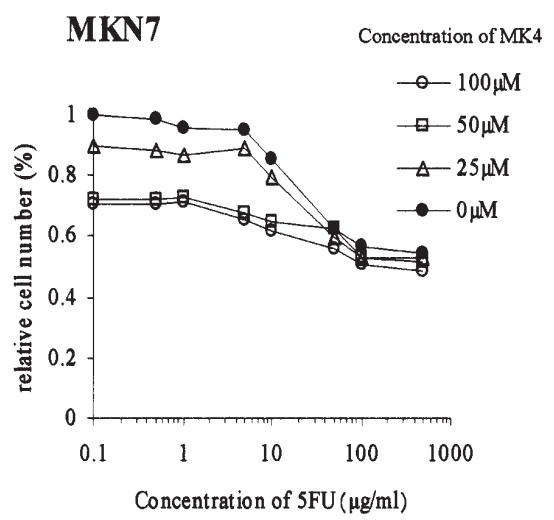

FU97

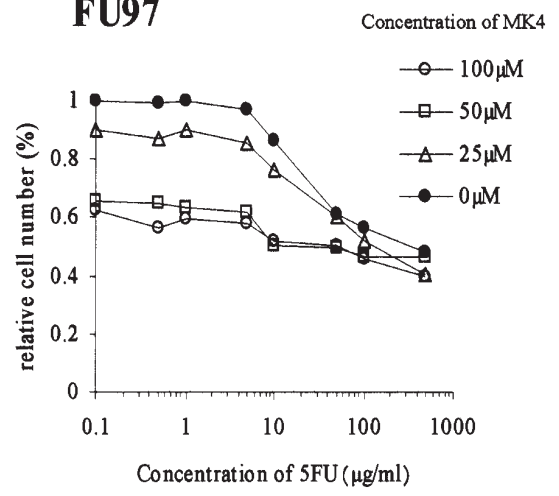

Figure 6. Effect of combined treatment of 5-fluorouracil (5-FU) plus MK4 on gastric cancer cell proliferation.

apoptosis and the enhanced effect of combined treatment with anticancer drugs on gastric cancer cells. In the present study, the following 4 kinds of cells arising from human gastric cancer were used: KATO III (signet ring cell carcinoma), MKN7 (highly-differentiated ring gland carcinoma), MKN74 (liver metastasis strain) and FU97 (AFP-producing gastric 

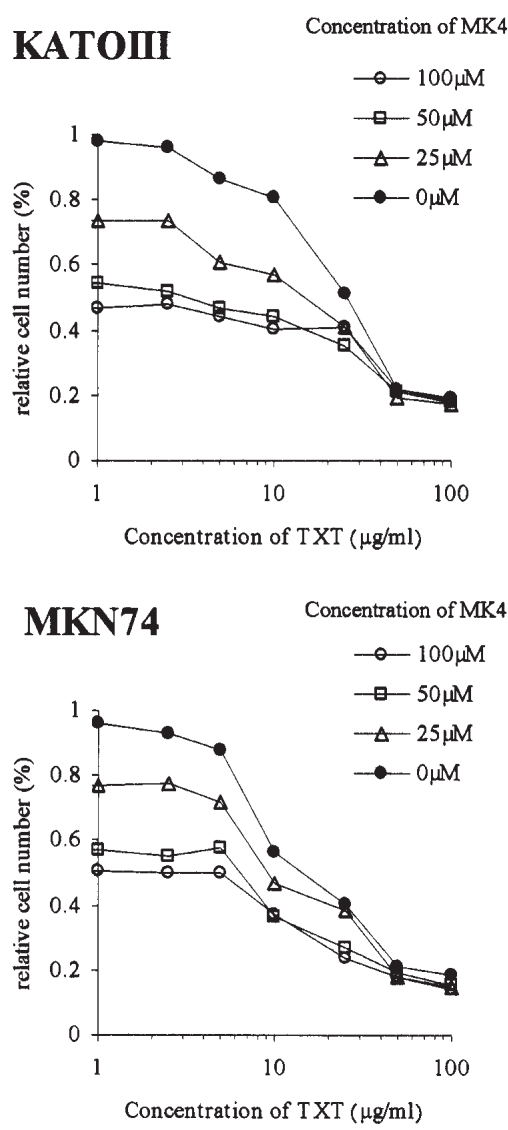
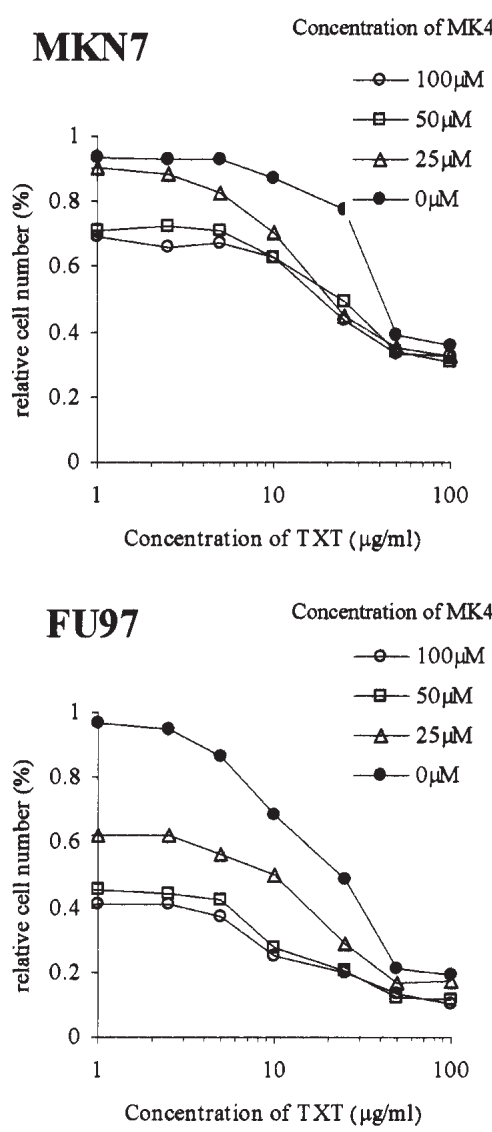

Figure 7. Effect of combined treatment of docetaxel (TXT) plus MK4 on gastric cancer cell proliferation.

cancer strain). MK4 was added at various concentrations and, after cells had been incubated for $96 \mathrm{~h}$ with $10 \mu \mathrm{M}$ or more of MK4, cell growth inhibition in a dose-dependent manner was recognized in each of the cell lines. The $\mathrm{ID}_{50}$ of these cell lines ranged from 25 to $50 \mu \mathrm{M}$, and these concentrations were slightly higher than the $\mathrm{ID}_{50}$ of leukemia cells, hepatocellular carcinoma cells or other cancerous tumors $(5,7)$. It was thus shown that MK4 is effective also in gastric cancer cells. Miyazawa et al reported that MK4 caused apoptotic changes including morphological narrowing of HL-60 cells or nuclear fragmentation, but with HL-60-bcl-2, where bcl-2 is expressed excessively, apoptosis did not take place and the differentiation inducing effect acting cytostatically was manifested (5). In this study, apoptosis was markedly induced in FU97 but, in the other cell lines, virtually no apoptotic changes were noted, although an induction of differentiation was exhibited. However, APO2.7 positive cells increased significantly in all of the cell lines and, in KATO III and FU97, activation of caspase-3 was recognized, indicating that apoptosis does occur in some of these cells. These findings suggested that there might be differences in the morphological mode of expression, depending on the type of cancer. On the other hand, Shibayama-Imazu et al reported that, in similar examinations of 2 kinds of gastric cancer cell (KATO III, NUGC-2), growth inhibition was recognized in each cell type with GGO but, with MK4, there was virtually no inhibitory effect (12). Yet, because their investigation covered only two cases and there are no other reports on MK4 in relation to gastric cancer cells, further studies are required.
Although the mechanism of cell growth inhibition by VK2 is little understood, it is brought about mainly by apoptosis and the induction of cell-cycle arrest. With regard to apoptosis, Nishimaki et al and Miyazawa et al reported that, with MDS or leukemia cells, a decrease of Bcl-2 expression by VK2 was recognized, together with the strengthening of BAX expression, depolarization of mitochondria membrane potential and activation of caspase-3, all of which indicated that apoptosis induction is suppressed significantly by caspase inhibition $(4,5)$. Shibayama-Imazu et al reported that, with TYK-nu ovarian cancer cells, caspase- 3 activity increased from the second day of exposure to VK2, and increases of DNA fragmentation and apoptotic cells were noted from the third day (12). In the present study, the APO2.7 used for detecting apoptotic cells by flow cytometry was a monoclonal antibody that reacts to 7A6 antigen, a mitrochondrial membrane protein. Because cells in the late stage of apoptosis shrink and are not detected by flow cytometry, APO2.7 identifies apoptotic cells at a relatively early stage $(14,21)$. In the present study, an exposure to $50 \mu \mathrm{M}$ of MK4 resulted in a statistically significant increase in the percentage of APO2.7 positive cells in each of the four cell lines as compared to controls. On the other hand, it was extremely interesting that caspase- 3 activity increased significantly in KATO III and FU97 compared to controls, but that no significant increase was recognized in MKN7 or MKN74. Clinically, signet ring cell carcinoma is an undifferentiated gastric cancer that stores mucus in the cytoplasm, and infiltrating growth and peritoneal seeding are its special characteristics $(22,23)$. AFP-producing gastric cancer is a 
special type of gastric cancer and synchronous or metachronous liver metastasis occurs in about $60 \%$ of such patients (24-26). Both KATO III and FU97 are derived from these cancers with a poor prognosis. On the contrary, tubular adenocarcinoma is a differentiated gastric cancer, and expansive growth and liver metastasis are its distinguishing characteristics, but its prognosis is relatively good (22). Although detailed mechanisms are obscure, these results indicate that the difference in caspase- 3 activity between differentiated gastric cancer cell lines and undifferentiated gastric cancer cell lines might be due to a difference in the apoptosis inducing capacity of MK4, depending on the special features of the cell strains.

With regard to cell-cycle arrest by VK2, Miyazawa et al reported that, with HL-60 leukemia cells and HL-60-bcl-2 cells, significant increases of G0/G1 phase and p27 $7^{\mathrm{KIP} 1}$, a cyclin-dependent kinase inhibitor, were manifested by MK4 exposure (5). Otsuka et al reported that, with PRF/PLC/5 and HepG2 hepatocellular carcinoma cells, protein kinase A (PKA), which regulates AP-2, USF-1, CREB and other transcription factors, becomes active under MK4 exposure (10). AP-2 and USF-1 are factors that inhibit tumor growth and metastasis $(27,28)$, and it is also known that PKA induces cell-cycle arrest, not only of G1 phase but also G2/M phase (29-31). Hitomi et al reported that, when using a mouse model in which PLC/PRF/5 hepatocellular carcinoma cells had been transplanted subcutaneously, administration of MK4 at $400 \mu \mathrm{M} /$ day manifested a significant inhibition of tumor growth as compared to controls (11). They also pointed out that there was a significant decrease in cyclin D1 and Cdk4 but not p16 in the models, and that such results suggested that G1 arrest of the cell cycle was induced by MK4. In our study, an increase of G0/G1 phase was observed in all four cell lines by exposure to 50 and $100 \mu \mathrm{M}$ of MK4 as compared to the controls, suggesting that G1 arrest of the cell cycle occurred. G0/G1 phase was approximately $70 \%$ in KATO III and FU97 but $80 \%$ or more in MKN7 and MKN74. These findings indicated that, as in the case of apoptosis, the influence of MK4 on the cell cycle varies between differentiated and undifferentiated gastric cancer cells.

Chemotherapy is one option for the treatment of advanced or recurrent cancer but, occasionally, it must be stopped because of side effects. This arises from the fact that many anticancer drugs damage not only tumor cells but normal cells as well. VK2, on the contrary, has virtually no side effects, its safety has been established and it exhibits an antitumor effect. Sakai et al reported that when MK4 and camptothecin, an anticancer drug, are used concurrently in human myeloid leukemia cells, extensive differentiation is induced as compared to when VK2 or capmtothecin are administered independently (2). Yokoyama et al reported that concurrent use of imatinib and VK2 in small cell lung cancer cell lines significantly induced apoptosis (32). In the present study, MK4 of various concentrations and two kinds of anticancer drug were administered to 4 gastric cancer cell lines and survival cell counts were determined. In each of the cancer cell lines, cell growth was inhibited in a dose-dependent manner by concomitant use of MK4 and an anticancer drug at up to $10 \mu \mathrm{M}$. These results indicate that such combinations can be used clinically and that the side effects of anticancer drugs can possibly be reduced by lowering dosages.

\section{Acknowledgements}

The authors are indebted to Professor J. Patrick Barron of the International Medical Communications Center of Tokyo Medical University for his review of this manuscript, and would like to thank Ms. Ayako Hirota, First Department of Internal Medicine, Tokyo Medical University, for her expert technical assistance.

\section{References}

1. Lamson DW and Plaza SM: The anticancer effects of vitamin K. Altern Med Rev 8: 303-318, 2003.

2. Sakai I, Hashimoto S, Yoda M, Hida T, Ohsawa S, Nakajo S and Nakaya K: Novel role of vitamin K2: a potent inducer of differentiation of various myeloid leukemia cell lines. Biochem Biophys Res Commun 205: 1305-1310, 1994.

3. Yaguchi M, Miyazawa K, Katagiri T, Nishimaki J, Kizaki M, Tohyama K and Toyama K: Vitamin K2 and its derivatives induce apoptosis in leukemia cells and enhance the effect of alltrans retinoic acid. Leukemia 11: 779-787, 1997.

4. Nishimaki J, Miyazawa K, Yaguchi M, Katagiri T, Kawanishi Y, Toyama K, Ohyashiki K, Hashimoto S, Nakaya K and Takiguchi T: Vitamin K2 induces apoptosis of a novel cell line established from a patient with myelodysplastic syndrome in blastic transformation. Leukemia 13: 1399-1405, 1999.

5. Miyazawa K, Yaguchi M, Funato K, Gotoh A, Kawanishi Y, Nishizawa Y, You A and Ohyashiki K: Apoptosis/differentiationinducing effects of vitamin $\mathrm{K} 2$ on HL-60 cells: dichotomous nature of vitamin K2 in leukemia cells. Leukemia 15: 1111-1117, 2001.

6. Wang Z, Nishikawa Y, Wang M and Carr BI: Induction of apoptosis via mitogen-activated protein kinase pathway by a $\mathrm{K}$ vitamin analog in rat hepatocytes. J Hepatol 36: 85-92, 2002 .

7. Nishikawa Y, Carr BI, Wang M, Kar S, Finn F, Dowd P, Zheng ZB, Kerns J and Naganathan S: Growth inhibition of hepatoma cells induced by vitamin $\mathrm{K}$ and its analogs. J Biol Chem 270: 28304-28310, 1995.

8. Osada S and Carr BI: Mechanism of novel vitamin K analog induced growth inhibition in human hepatoma cell line. J Hepatol 34: 676-682, 2001.

9. Wang Z, Wang M, Finn F and Carr BI: The growth inhibitory effects of vitamins $K$ and their actions on gene expression. Hepatology 22: 876-882, 1995.

10. Otsuka M, Kato N, Shao RX, Hoshida Y, Ijichi H, Koike Y, Taniguchi H, Moriyama M, Shiratori Y, Kawabe T and Omata M: Vitamine K2 inhibits the growth and invasiveness of hepatocellular carcinoma cells via protein kinase A activation. Hepatology 40: 243-251, 2004.

11. Hitomi M, Yokoyama F, Kita Y, Nonomura T, Masaki T, Hoshiji H, Inoue H, Kinekawa F, Kurokohchi K, Uchida N, Watanabe D and Kuriyama S: Antitumor effects of vitamins K1, $\mathrm{K} 2$ and $\mathrm{K} 3$ on hepatocellular carcinoma in vitro and in vivo. Int J Oncol 26: 713-720, 2005.

12. Shibayama-Imazu T, Sakairi S, Watanabe A, Aiuchi T, Nakajo S and Nakaya K: Vitamine K2 selectively induced apoptosis in ovarian TYK-nu and pancreatic MIA PaCa-2 cells out of eight solid tumor cell lines through a mechanism different from geranylgeraniol. J Cancer Res Clin Oncol 129: 1-11, 2003.

13. Fujita H, Tomiyama J and Tanaka T: Vitamin K2 combined with all-trans retinoic acid induced complete remission of relapsing acute promyelocytic leukaemia. Br J Haematol 103: 584-585, 1998.

14. Yaguchi M, Miyazawa K, Otawa M, Katagiri T, Nishimaki J, Uchida Y, Iwase O, Gotoh A, Kawanishi Y and Toyama K: Vitamin K2 selectively induces apoptosis of blastic cells in myelodysplastic syndrome: flow cytometric detection of apoptotic cells using APO2.7 monoclonal antibody. Leukemia 12: 1392-1397, 1998 .

15. Takami A, Nakao S, Ontachi Y, Yamauchi H and Matsuda T: Successful therapy of myelodysplastic syndrome with menatetrenone, a vitamin K2 analog. Int J Hematol 69: 24-26, 1999.

16. Habu D, Shiomi S, Tamori A, Takeda T, Tanaka T, Kubo S and Nishiguchi S: Role of vitamin K2 in the development of hepatocellular carcinoma in women with viral cirrhosis of the liver. JAMA 292: 358-361, 2004. 
17. Venugopal M, Jamison JM, Gilloteaux J, Koch JA, Summers M, Giammar D, Sowick C and Summers JL: Synergistic antitumor activity of vitamins $\mathrm{C}$ and $\mathrm{K} 3$ on human urologic tumor cell lines. Life Sci 59: 1389-1400, 1996.

18. Noto V, Taper HS, Yi-Hua J, Janssens J, Bonte J and Loecker W: Effects of sodium ascorbate (vitamin C) and 2-methyl-1,4naphthoquinone (vitamin K3) treatment on human tumor cell growth in vitro. Cancer 63: 901-906, 1989.

19. Ohizumi H, Masuda Y, Nakajo S, Sakai I, Ohsawa S and Nakaya K: Geranylgeraniol is a potent inducer of apoptosis in tumor cells. J Biochem 117: 11-13, 1995.

20. Orimo H, Shiraki M, Tomita A, Morii H, Fujita T and Ohata M: Effects of menatetrenone on the bone and calcium metabolism in osteoporosis: a double-blind placebo-controlled study. J Bone Miner Metab 16: 106-112, 1998.

21. Koester SK, Roth P, Mikulka WR, Schlossman SF, Zhang C and Bolton WE: Monitoring early cellular responses in apoptosis is aided by the mitochondrial membrane protein-specific monoclonal antibody APO2.7. Cytometry 29: 306-312, 1997.

22. Adachi Y, Yasuda K, Inomata M, Sato K, Shiraishi N and Kitano S: Pathology and prognosis of gastric carcinoma: well versus poorly differentiated type. Cancer 89: 1418-1424, 2000.

23. Yang XF, Yang L, Mao XY, Wu DY, Zhang SM and Xin Y: Pathological behavior and molecular mechanism of signet ring cell carcinoma and mucinous adenocarcinoma of the stomach: a comparable study. World J Gastroenterol 10: 750-754, 2004.

24. Takahashi Y, Ohta T and Mai M: Angiogenesis of AFP producing gastric carcinoma: correlation with frequent liver metastasis and its inhibition by anti-AFP antibody. Oncol Rep 11: 809-813, 2004.
25. Kono K, Amemiya H, Sekikawa T, Iizuka H, Takahashi A, Fujii $\mathrm{H}$ and Matsumoto $\mathrm{Y}$ : Clinicopathological features of gastric cancers producing alpha-fetoprotein. Dig Surg 19: 359-365, 2002.

26. Iida M, Imura J, Furuichi T, Sawada T, Nagawa $H$ and Fujimori T: Alteration of the AT motif binding factor- 1 expression in $\alpha$-fetoprotein producing gastric cancer: is it an event for differentiation and proliferation of the tumors? Oncol Rep 11: 3-7, 2004.

27. Zeng YX, Somasundaram K and El-Deiry WS: AP2 inhibits cancer cell growth and activates p21WAF1/CIP1 expression. Nat Genet 15: 78-82, 1997.

28. Luo X and Sawadogo M: Antiproliferative properties of the USF family of helix-loop-helix transcription factors. Proc Natl Acad Sci USA 93: 1308-1313, 1996.

29. Dumaz N, Light Y and Marais R: Cyclic AMP blocks cell growth through Raf-1-dependent and Raf-1-independent mechanisms. Mol Cell Biol 22: 3717-3728, 2002.

30. Duckworth BC, Weaver JS and Ruderman JV: G2 arrest in Xenopus oocytes depends on phosphorylation of cdc 25 by protein kinase A. Proc Natl Acad Sci USA 99: 16794-16799, 2002.

31. McKenzie FR and Pouyssegur J: cAMP-mediated growth inhibition in fibroblasts is not mediated via mitogen-activated protein (MAP) kinase (ERK) inhibition: cAMP-dependent protein kinase induces a temporal shift in growth factor-stimulated MAP kinases. J Biol Chem 271: 13476-13483, 1996.

32. Yokoyama T, Miyazawa K, Yoshida T and Ohyashiki K: Combination of vitamin K2 plus imatinib mesylate enhances induction of apoptosis in small cell lung cancer cell lines. Int J Oncol 26: 33-40, 2005. 